Kell, Adolf

\title{
Grenzgänge, Traditionen und Zukünfte in der Deutschen Gesellschaft für Erziehungswissenschaft. Kongresse zur Reflexion - auch für die Sektion Berufs- und Wirtschaftspädagogik? \\ Erziehungswissenschaft 25 (2014) 49, S. 49-64
}

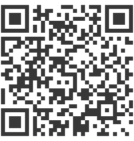

Quellenangabe/ Reference:

Kell, Adolf: Grenzgänge, Traditionen und Zukünfte in der Deutschen Gesellschaft für Erziehungswissenschaft. Kongresse zur Reflexion - auch für die Sektion Berufs- und Wirtschaftspädagogik? - In: Erziehungswissenschaft 25 (2014) 49, S. 49-64 - URN: urn:nbn:de:0111-pedocs-100819 - DOI: 10.25656/01:10081

https://nbn-resolving.org/urn:nbn:de:0111-pedocs-100819

https://doi.org/10.25656/01:10081

in Kooperation mit / in cooperation with:

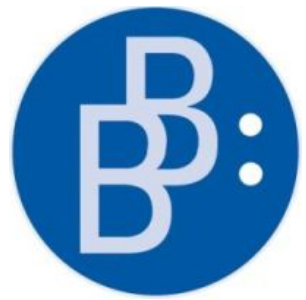

https://www.budrich.de

\section{Nutzungsbedingungen}

Gewährt wird ein nicht exklusives, nicht übertragbares, persönliches und beschränktes Recht auf Nutzung dieses Dokuments. Dieses Dokument ist ausschließlich für den persönlichen, nicht-kommerziellen Gebrauch bestimmt. Die Nutzung stellt keine Übertragung des Eigentumsrechts an diesem Dokument dar und gilt vorbehaltlich der folgenden Einschränkungen: Auf sämtlichen Kopien dieses Dokuments müssen alle Urheberrechtshinweise und sonstigen Hinweise auf gesetzlichen Schutz beibehalten werden. Sie dürfen dieses Dokument nicht in irgendeiner Weise abändern, noch dürfen Sie dieses Dokument für öffentliche oder kommerzielle Zwecke vervielfältigen, öffentlich ausstellen, aufführen, vertreiben oder anderweitig nutzen.

Mit der Verwendung dieses Dokuments erkennen Sie die Nutzungsbedingungen an.

\section{Terms of use}

We grant a non-exclusive, non-transferable, individual and limited right to using this document.

This document is solely intended for your personal, non-commercial use. Use of this document does not include any transfer of property rights and it is conditional to the following limitations: All of the copies of this documents must retain all copyright information and other information regarding legal protection. You are not allowed to alter this document in any way, to copy it for public or commercial purposes, to exhibit the document in public, to perform, distribute or otherwise use the document in public.

By using this particular document, you accept the above-stated conditions of use.

\section{Kontakt / Contact:}

peDOCS

DIPF | Leibniz-Institut für Bildungsforschung und Bildungsinformation Informationszentrum (IZ) Bildung

E-Mail: pedocs@dipf.de

Internet: www.pedocs.de

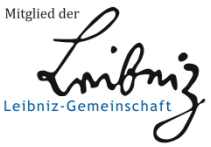




\section{Erziehungswissenschaft}

Mitteilungen der Deutschen Gesellschaft

für Erziehungswissenschaft (DGfE)

Heft 49

25. Jahrgang 2014

ISSN 0938-5363

Verlag Barbara Budrich 


\section{INHALTSVERZEICHNIS}

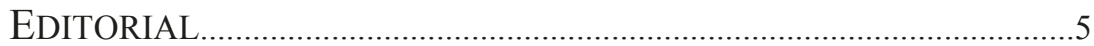

BEITRÄGE ZUM THEMA ,ERINNERUNGSKULTUREN PREISE, NAMEN UND DISZIPLINPOLITIK“

Stellungnahme des Vorstandes zur Rücknahme des Namens für den

Forschungspreis vom 13. Januar 2014

Andreas Hoffmann-Ocon

Heinrich Roth und der Preis der Forschung - Historisierungen,

Verundeutlichungen und erziehungswissenschaftliche

Erinnerungsgeschichten

Benjamin Hasselhorn

Erinnerung im Streit. Zum Umgang der deutschen

Geschichtswissenschaft mit ihrer eigenen Vergangenheit

Eva Matthes \& Carola Groppe

Historische Bildungsforschung und Erinnerungskultur. Eine

Stellungnahme der Sektion Historische Bildungsforschung.

Daniel Tröhler

Wissenschaftliche Ehrungen oder der doppelte Gestus der

Auszeichnung. Bemerkungen zur fallengelassenen Idee eines Heinrich

Roth-Forschungspreises der DGfE

Jörg Schlömerkemper

„Nomen est omen“ - oder geht es auch ohne? Was kann/Was soll der

Name einer Person bei einem Preis bedeuten? Anmerkungen nach dem

Streit um die Benennung des Forschungspreises der DGfE nach

Heinrich Roth

BEITRÄGE

Adolf Kell

Grenzgänge, Traditionen und Zukünfte in der Deutschen Gesellschaft für Erziehungswissenschaft. Kongresse zur Reflexion - auch für die Sektion Berufs- und Wirtschaftspädagogik? 


\section{MitTEILUNGEN DES VORSTANDS}

Protokoll der Mitgliederversammlung der Deutschen Gesellschaft für

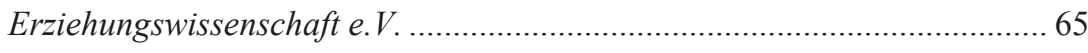

Ergebnis der Wahl zum Vorsitz und Vorstand der DGfE............................ 71

Liste der unbekannt verzogenen Mitglieder ............................................... 72

BERICHTE AUS DEN SEKTIONEN

Sektion 1 - Historische Bildungsforschung ............................................... 75

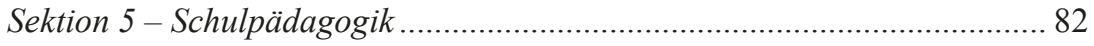

Sektion 8 - Sozialpädagogik und Pädagogik der frühen Kindheit................ 85

Sektion 9 - Erwachsenenbildung .............................................................. 91

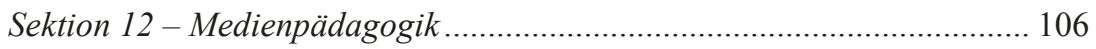

Sektion 13 - Differentielle Erziehungs- und Bildungsforschung ................ 108

NOTIZEN

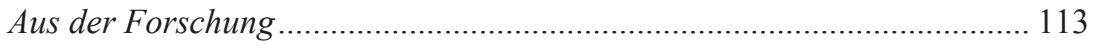

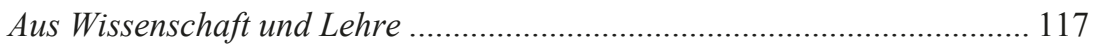

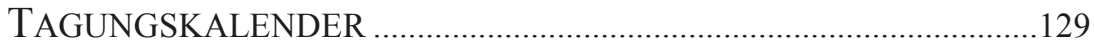




\section{BEITRÄGE}

\section{Grenzgänge, Traditionen und Zukünfte in der Deutschen Gesellschaft für Erziehungswissenschaft}

Kongresse zur Reflexion - auch für die Sektion Berufs- und Wirtschaftspädagogik?

Adolf Kell

\section{Kongresse der Deutschen Gesellschaft für Erziehungswissenschaft: Entwicklungen und Strukturen.}

Die Kongresse der Deutschen Gesellschaft für Erziehungswissenschaft (DGfE) haben sich von kleinen wissenschaftlichen Arbeitstagungen im Kontext der alle zwei Jahre durchgeführten Mitgliederversammlungen mit einigen Referaten zu grundlegenden und aktuellen Problemen in Erziehung und Bildung zu Großveranstaltungen dieser wissenschaftlichen Fachgesellschaft mit über 2.000 Teilnehmenden entwickelt (vgl. Berg/Herrlitz/Horn 2004). Inzwischen hat sich eine mehrfach erprobte Organisationsstruktur bewährt (vgl. DGfE 2014: 352f.), die es ermöglichen soll, zu einem Rahmenthema differenzierte Beiträge von Mitgliedern der Sektionen, Kommissionen und Arbeitsgruppen vorzutragen und darüber innerwissenschaftlich zu diskutieren, aber auch Gastbeiträge von Vertretern benachbarter Wissenschaften einzubeziehen in interdisziplinäre Diskurse.

Die von den jeweiligen Vorständen beschlossenen Titel für die Kongresse, mit denen Rahmenthemen kurz gefasst umrissen sind (vgl. Berg/Herrlitz/ Horn 2004: insb. 93ff.), können interpretiert werden als Reaktionen einerseits auf gesellschaftliche Entwicklungen und Problemlagen, die Erziehung und Bildung besonders beeinflussen, und andererseits auf innerwissenschaftliche Entwicklungen in den Sektionen, Kommissionen und Arbeitsgruppen, die auf bestimmte gesellschaftliche Problemlagen in spezifischer Wiese reagieren und dadurch die Differenzierungen innerhalb der DGfE beeinflussen (vgl. Behm 2014). Daraus folgt als Daueraufgabe für die Organisation und Strukturierung der Wissenschaftlichen Fachgesellschaft und ihrer Kongresse, u.a. die Spannungen zwischen Einheit und Differenz auszuhalten und zu gestalten.

„Die Veranstaltung von Fachkongressen, die alle zwei Jahre stattfinden“, wird in der Satzung als erste Aufgabe genannt, um den Zweck der DGfE zu 
erfüllen. Deshalb sind mit der Wahl von Titeln, Rahmenthemen, Leitformeln etc. für die Kongresse durch den Vorstand auch steuernde Absichten verbunden. Die Steuerungsmöglichkeiten des Vorstandes sind jedoch begrenzt, weil die wissenschaftliche Arbeit von den Mitgliedern geleistet wird und nur auf freiwilliger Basis koordiniert werden kann, vor allem durch die Zusammenarbeit in den Sektionen, Kommissionen und Arbeitsgruppen der DGfE. Allerdings kann der Vorstand in Zusammenarbeit mit dem lokalen Organisationskomitee die Bearbeitung des Rahmenthemas durch die Organisation des Kongresses beeinflussen, zum Beispiel durch die Auswahl von Referenten und Referentinnen für Einleitungs- und Parallelvorträge und Vereinbarungen über die Themenbearbeitung, durch Initiierung von Symposien, Arbeitsgruppen, Workshops etc. und durch personelle Beteiligung an der Planung und Durchführung, durch Review-Verfahren und durch die Zuteilung von Zeitkontingenten für verschiedene Veranstaltungsformen. ${ }^{1}$ Die grundsätzliche Schwierigkeit bei der Bewältigung dieser Steuerungsaufgabe besteht darin, eine Balance $\mathrm{zu}$ finden zwischen (vorrangiger) Button-up-Koordination und (nachrangiger) Top-down-Steuerung.

Die Titel der 23. und 24. DGfE-Kongresse „Erziehungswissenschaftliche Grenzgänge - Markierungen und Vermessungen“" (2012 in Osnabrück) und „Traditionen und Zukünfte“ (2014 in Berlin) signalisieren Bedarfe nach Reflexion und Verständigung sowohl über die eigene Wissenschaft und ihrer Organisation als auch über die Beziehungen zu anderen Wissenschaften und den Möglichkeiten und Grenzen interdisziplinärer Zusammenarbeit (vgl. Müller/Bohne/Thole 2013). Zu der Frage, ob und inwieweit vor dem Hintergrund der kurz umrissenen Steuerungsproblematik die Rahmenthemen dieser beiden Kongresse beachtet und bearbeitet wurden, sollen im Folgenden einige subjektive Wahrnehmungen und Eindrücke erläutert werden, und zwar mit Bezug auf die Themen, die in den Programmheften für diese beiden Kongresse von Mitgliedern der Sektion Berufs- und Wirtschaftspädagogik (BWP) angeboten wurden. Bei der Beobachtung der beiden DGfE-Kongresse hatte ich zunächst das vage Gefühl, dass die Sektion BWP an der Reflexion über ihre eigene wissenschaftliche Arbeit vergleichsweise wenig interessiert ist. Dieses Gefühl hat mich veranlasst, etwas genauer hinzusehen.

Der Rechenschaftsbericht des DGfE-Vorstandes über die Vorstandsperiode 2012 bis 2014 enthält reflektierende Aussagen über die Organisation des Kongresse zum50-jährigen Jubiläum 2014 (In: Erziehungswissenschaft 25, 48: 146 f.). 


\section{Beiträge aus der Sektion Berufs- Wirtschaftspädagogik zu den Kongressen}

Sicherlich konnte und kann nicht erwartet werden, dass alle Beiträge, die für die Kongresse angemeldet und zugelassen werden, das jeweilige Rahmenthema beachten. Denn die berechtigten Bedürfnisse von Autorinnen und Autoren nach Darstellung der je eigenen wissenschaftlichen Arbeit und nach Diskussionen in Expertenkreisen sind (auch oder vorrangig?) zu berücksichtigen. Die Vorstände und die LOKs sind offenbar mit diesen Bedürfnissen auch weitgehend weise und tolerant umgegangen, wenn man die Programmhefte, Kongressberichte und weitere zahlreiche Publikationen über die Kongressbeiträge überblickt. $\mathrm{Zu}$ erwarten ist aber ein Mindestmaß an Berücksichtigung der Rahmenthemen durch die einzelnen Sektionen, Kommissionen und Arbeitsgruppen der DGfE und ihrer Mitglieder. Für die Sektion BWP vermisse ich dieses Mindestmaß.

Die Beiträge, die in beiden Kongressprogrammen von Mitgliedern der Sektion BWP oder von anderen Autorinnen und Autoren angekündigt wurden, die thematisch mit Bezug auf die gesellschaftliche Praxis „Berufsbildung“" wissenschaftlich arbeiten (zur Abgrenzung vgl. Buer van/Kell 1999: insbesondere 52; Kell 2010) und/oder die dem Erkenntnisgegenstand der BWP zuzuordnen sind, ermöglichen eine Einschätzung, ob und inwieweit die Autorinnen und Autoren die beiden Rahmenthemen in den Ankündigungen ihrer Beiträge berücksichtigt haben. Dazu folgende quantitative Übersicht (an erster Stelle sind jeweils die Anzahl aller Beiträge aufgeführt, an zweiter Stelle die Beiträge aus dem oben umrissenen Autorenkreis):

Abbildung: Anteile der Beiträge aus dem Themenbereich Berufs- und Wirtschaftspädagogik an den Veranstaltungsangboten der DGfE-Kongresse 2012 und 2014

\begin{tabular}{lccc} 
& 23.Kongress & 24. Kongress & Gesamt \\
\hline $\begin{array}{l}\text { Vorprogramme } \\
\text { (sonntags) }\end{array}$ & $20 / 0$ & $23 / 0$ & $43 / 0$ \\
\hline Eröffnungsvorträge & $1 / 0$ & $1 / 0$ & $2 / 0$ \\
\hline Parallelvorträge & $8 / 0$ & $12 / 0$ & $20 / 0$ \\
\hline Symposien & $25 / 2$ & $45 / 1$ & $70 / 3$ \\
\hline Arbeitsgruppen & $56 / 5$ & $75 / 6$ & $131 / 11$ \\
\hline Themenforen & $11 / 0$ & $7 / 0$ & $18 / 0$ \\
\hline Forschungsforen & $30 / 2$ & $32 / 0$ & $62 / 2$ \\
\hline Postersession & $79 / 7$ & $160 / 11$ & $239 / 18$ \\
\hline Podiumsdiskussionen & $2 / 0$ & $-/-$ & $2 / 0$ \\
\hline $\begin{array}{l}\text { Wissenschaftliche Streit- } \\
\text { gespräche }\end{array}$ & $1 / 0$ & $1 / 0$ & $2 / 0$ \\
\hline
\end{tabular}

Quelle: eigene Darstellung 
Von den 589 angekündigten Aktivitäten entfallen 34 (knapp sechs Prozent) auf den oben umrissenen Autorenkreis. Die DGfE hat ca. 3000 und die Sektion BWP gut 300 Mitglieder. $^{2}$ Quantitativ waren die Mitglieder der Sektion BWP bezogen auf diese Relation bei den Kongress-Aktivitäten unterrepräsentiert. $^{3}$

Zum thematischen Spektrum der 34 angekündigten Beiträge fällt Folgendes auf: Zwei dominante Themenkomplexe können als Reaktionen auf bildungspolitische Probleme interpretiert werden: (1) Kompetenzdiagnose, -messung und -entwicklung (neun Beiträge, $26 \%)^{4}$;(2) Berufsorientierung und -vorbereitung; Übergänge, insbesondere an der ersten Schwelle zwischen Schule und Berufsausbildung/Erwerbsarbeit (19 Beiträge, 56\% $)^{5}$. Hinzu kommen vier Beiträge zur Didaktik $(12 \%)^{6}$ und zwei sonstige $(6 \%)^{7}$.

Die inhaltlichen Bezüge zu den Kongressthemen sind äußerst spärlich. Zum Rahmenthema Grenzgänge sind bei fünf von 16 Beiträgen zumindest sprachliche und inhaltliche Bezüge zu erkennen. ${ }^{8}$ Zum Rahmenthema Traditionen und Zukünfte ist allenfalls ein Beitrag einschlägig. ${ }^{9}$

\section{Einige Anregungen zum Nachdenken}

\subsection{Begrenzte gemeinsame Selbstreflexionen in der Sektion}

Die von den beiden letzten DGfE-Kongressen angeregten Selbstreflexionen hatten bei der Sektion BWP zunächst keine sichtbare Resonanz. Insofern besteht ein gewisser Nachholbedarf. Er ist auch daran zu erkennen, dass in den letzten Jahren keine Sektionstagungen stattgefunden haben, die auf Bilanzierungen der eigenen wissenschaftlichen Arbeit in Theorie und Praxis fokus-

Auskunft des DGfE-Büros am 05. Mai 2014: 2285 ordentliche Mitglieder, 786 assoziierte und 87 sonstige Mitglieder; das Adressbuch der Sektion BWP enthält 302 Eintragungen.

3 Von den 34 Aktivitäten entfallen 13 auf Autorinnen und Autoren, die nicht Mitglieder der Sektion BWP sind (2012: P 3, 19, 72; 2014: P 53, 54, 57, 59, 60, 62, 63, 68, 69, 131 - alle mit Beiträgen zum Themenkomplex 2). 2012=3: AG 12, P 49, 52; $2014=6$ : SY 12, AG 1, $25,47,62, \mathrm{P} 49$.

$42012=3$ : AG 12, P 49, 52; 2014=6: SY 12, AG 1, 25, 47, 62, P 49.

52012 = 9: AG 21, 43, 48, FF 10, FF 26, P 3, 19, 42, 72; 2014=10; AG 57, P 53, 57, 59, 60, $62,63,68,69,131$.

$6 \quad 2012=3$ : SY 14 und 17, P 11; 2014=1: P 54.

7 2012: AG 53 (Erfolgsfaktoren beruflicher Bildung); 2014: AG 51 (Design-Based Research - ein Forschungsparadigma für die Bildungswissenschaften?).

8 SY 14: Am Beispiel von Entrepreneurship Education wird ein Grenzgang zwischen Erziehungswissenschaft und Ökonomie thematisiert. SY 17 thematisiert Entgrenzungen in und von Bildungsinstitutionen. AG 48 thematisiert biographische Grenzgänge im Übergangssystem. AG 53 verweist auf Grenzverschiebungen in der beruflichen Bildung. FF 26.

AG 51 Design-Based Research als ein Forschungsparadigma für die Bildungswissenschaften. 
siert waren. Zwar ist in der berufsbildungswissenschaftlichen Forschung und Lehre an den Universitäten ein beachtliches Niveau an theoretischen Begründungen für die je eigene wissenschaftliche Arbeit zu erkennen, es liegen zahlreiche Beiträge zu Standortbestimmungen und eigenen Positionierungen vor und die in den letzten Jahren erschienen Handbücher enthalten auch zahlreiche Beiträge zur Wissenschaftsgeschichte, zu Theorieentwicklungen, Forschungskonzepten etc. (vgl. Huisinga/Lisop 1999; Sloane/Twardy/Buschfeld 2004; Rauner 2005; Nickolaus/Pätzold/Reinisch/Tramm 2010; Niedermair 2013), aber durch Tagungen ermöglichte direkte Diskurse sowie gemeinsame Bilanzierungen fehlen.

Der letzte Versuch, durch einen Sammelband mit Beiträgen zum „Selbstverständnis der Disziplin Berufs- und Wirtschaftspädagogik“ (Büchter/Klusmeyer/Kipp 2009) einen Diskurs anzuregen, hat keine erkennbare Resonanz gefunden. Dieser Versuch hat auch nicht zu einschlägigen Beiträgen auf den beiden DGfE-Kongressen 2012 und 2014 angeregt. Ein weiterer wichtiger Anlass, die berufsbildungswissenschaftliche Theorieentwicklung bis in die Gegenwart zu verfolgen, bietet Jürgen Zabecks umfangreiche „Geschichte der Berufserziehung und ihrer Theorie“ (Zabeck 2013). In seinen abschließenden Bemerkungen hat er nachvollziehbar begründet, warum er die Geschichte der Theorieentwicklung nicht bis in seine - 2014 beendete - Gegenwart verfolgt hat und es jüngeren Kolleginnen und Kollegen überlässt, sich seines Themas anzunehmen und die zeitliche Lücke zu schließen. Ein aktueller Diskurs in der Sektion BWP über ihr Selbstverständnis stünde auch im Einklang mit seinem Vermächtnis.

Solche eher innerdisziplinären Anstöße zur Selbstreflexion werden von außen verstärkt durch eine bedeutende Veränderung in der Gewichtung politischer Ziele: Nach mehreren internationalen Vereinbarungen (vgl. Deutsche UNESCO-Kommission 2009: 9f.) sind in der Europäischen Union und in Deutschland rechtlich verbindliche Rahmen geschaffen worden, um das globale Ziel Inklusion durch Planung und Gestaltung in den gesellschaftlichen Subsystemen und auf allen Ebenen erreichbar zu machen. Dabei wird von der Inklusion im Bildungssystem ein wichtiger Beitrag zur Zielerreichung erwartet. Auf Grund der Bedeutung der Berufsbildung im Bildungssystem und für Entwicklungen im Beschäftigungssystem sind die Berufsbildungspraxis und ihre Theorie besonders herausgefordert. Die BWP darf aber nicht nur (nachgängig) auf die daraus resultierenden Veränderungen in der Berufsbildungspraxis reagieren, sondern sie sollte sich (vorausschauend auf der Makroebene) mit der Zielkategorie Inklusion auseinander setzen und sowohl über die Beziehungen zur Zielkategorie Berufsbildung als auch darüber reflektieren, welches Selbstverständnis (Einstellungen, Haltungen, Beliefs etc.) sie dem gesamten pädagogischen Personal in der Berufsbildung durch deren Berufsbildung vermitteln will, z. B. durch curriculare Konstruktionen von Berufsbildungsgängen, die am Ziel Inklusion orientiert sind. 
Angesichts der Bedeutung, die Einstellungen und Haltungen des Personals für ihr pädagogisches Handeln haben, rückt die Frage in den Vordergrund, welche am Inklusionsziel orientierte Einstellungen und Haltungen durch Professionalisierungsprozesse in den Berufsbildungsgängen vermittelt werden sollen. Und diese Frage kann nur mit Bezug auf die Selbstverständnisse der Wissenschaften beantwortet werden, die in transdisziplinärer Kooperation zur Erreichung des Inklusionsziels beitragen wollen und können. Denn um „Inklusive Bildung professionell gestalten“ zu können (Döbert/Weishaupt 2013), muss die Berufsbildung des gesamten Personals in der Berufsbildung auf die Zielkategorie Inklusion orientiert werden.

In allen vom Bundesministerium für Bildung und Forschung initiierten Expertisen zur inklusiven Bildung, die in Döbert/Weishaupt (2013) publiziert sind, wird hervorgehoben, dass vor allem die Einstellungen, Haltungen etc. für Zielerreichungen große Bedeutung haben und deshalb vorrangig auf das Inklusionsziel orientiert werden müssen. Und durch eine empirische Studie im Bundesinstitut für Berufsbildung ist insbesondere mit Bezug auf die pädagogischen Fachkräfte im „Übergangssystem“ bestätigt worden: „Die Fähigkeit zur (Selbst-)Reflexion stellt eine bedeutende Dimension von Professionalität dar" (Bylinski 2014: 253, Hervorhebung im Original).

Individuelle Reflexionen über die eigene (Berufs)Biographie und kollektive Reflexionen über die Geschichte der eigenen Disziplin und ihr Selbstverständnis sind also wichtige Voraussetzungen für erfolgreiche Berufsbildungsprozesse des Personals, durch deren praktisches Handeln das Ziel Inklusion in der Berufsbildung erreicht werden soll. Deshalb erfordert auch die politisch und rechtlich vorgegebene Zielkategorie Inklusion eine Reflexion über das Selbstverständnis der BWP.

Von diesem Selbstverständnis ist auch abhängig, wie (berufsbildungswissenschaftlich fundierte) Professionalität als Zielperspektive interpretiert wird und wie Professionalisierung durch Lernen und Arbeiten in berufsbezogenen Bildungsgängen gestaltet werden sollen, um zur Erreichung des Inklusionsziels beitragen zu können. Wenn zur Zielerreichung „Entwicklungen hin $\mathrm{zu}(\mathrm{r})$ multiprofessionellen Organisationen, zur vernetzten Kooperation in Zivilgesellschaften, zum multiprofessionellen Management" erkennbar und erforderlich sind und sich in Lernenden Regionen pädagogische Handlungsbereiche stärker vernetzen, dann sind „multi-professionells“ gefordert (Tippelt/Schmidt-Hertha 2013: 214), die beruflich gebildet werden müssen in der Spannung von Gemeinsamem und Besonderem (von Einheit und Differenz). Sowohl zur Bestimmung des Gemeinsamen als auch zur Gestaltung der Beziehungen zum Besonderen anderer Wissenschaften und deren Professionali- 
täts-Perspektiven ist ein konsensiertes Selbstverständnis der BWP erforderlich. $^{10}$

Die Integration der nachwachsenden Generationen in die Gesellschaft gelingt durch die Berufsbildung in den gegenwärtigen Strukturen des (Berufs-) Bildungssystems ${ }^{11}$ nicht vollständig. Dass die Integrationsleistungen des Berufsbildungssystems eher nachgelassen haben, ist in den nationalen Bildungsberichten dokumentiert und insbesondere an der Entwicklung des so genannten „Übergangssystems“ zu erkennen. Theoretisch strittig ist, ob und inwieweit politische und pädagogische Aktivitäten, die am Beruf als zentrale Dimension der Zielkategorie Berufsbildung orientiert sind, inklusiomsförderlich oder inklusionshemmend wirken und welcher Begriff von Beruf geeignet sein könnte, zur Erreichung des Inklusionsziels beizutragen (vgl. Kell 2013). Zur Reflexion über das Selbstverständnis der BWP ist deshalb auch eine Reflexion über ihren Berufsbegriff unumgänglich.

Vom Ergebnis einer theoretischen Reflexion über den Beruf als Zieldimension in der Zielkategorie Inklusion und eine klare berufsbildungswissenschaftliche Begriffsbestimmung hängen auch Entscheidungen und Optionen zur pädagogischen Professionalität des Personals in der Berufsbildung ab. Unbestritten ist der große Einfluss des pädagogischen Personals auf die Entwicklungen der lernenden und arbeitenden Subjekte im dualen System, im Berufsschul- und im Übergangssystem, aber auch im Weiterbildungs- und Beschäftigungssystem - unter dem Einfluss der jeweiligen systemischen Umwelten. Klärungs- und entscheidungsbedürftig ist die Frage, mit welchen Berufsbildungsgängen, welches Personal für welche Arbeitsplätze/Berufe ausund weitergebildet und wie ein (neues) Segment des Berufsbildungssystems für das gesamte Personal politisch gestaltet werden soll und kann, das dem Inklusionsziel durch Sicherung von Durchlässigkeit und Chancengleich auch in diesem Segment des Berufsbildungssystems entspricht (vgl. Buchmann/ Bylinski 2013).

Dafür sind auch Klärungen der Wechselbeziehungen zwischen der BWP zur Schul-, Sozial- und Sonderpädagogik und auch zu den Fachdidaktiken erforderlich und sind deren Berufs/Professionsverständnisse und deren Berufsbildungsgänge im Schul- und Hochschulsystem zu berücksichtigen. Die praktische Relevanz (un)geklärter Wechselbeziehungen zwischen diesen erziehungswissenschaftlichen Teildisziplinen ist an den Beiträgen zum 24. DGfEKongress zu erkennen: Von den 19 Beiträgen zum Themenbereich (2) (Übergangssystem) wurden 13 von Autorinnen und Autoren eingebracht, die nicht

$10 \mathrm{Zu}$ klären wäre unter anderem, welche Alleinstellungsmerkmale von der BWP in einer transdisziplinären Kooperation geltend gemacht werden können und sollen.

11 Von der vorberuflichen Bildung bis zur beruflichen/wissenschaftlichen Weiterbildung (vgl. Kell 2006: 453). 
Mitglieder der Sektion BWP sind (siehe oben und FN 9). ${ }^{12}$ Reflexionen über solche Wechselbeziehungen setzen aber ein begründetes Selbstverständnis voraus, über das (auch) in der Sektion ein Konsens erarbeitet werden sollte.

Als ein Beispiel gemeinsamer Reflexion und Verständigung in der Sektion BWP kann auf das „Basiscurriculum für das universitäre Studienfach Berufs- und Wirtschaftspädagogik“" (Sektion BWP 2003) hingewiesen werden. Die aktualisierte Fassung (Sektion BWP 2014) soll auch in der Zukunft weiter entwickelt werden. ${ }^{13}$ Dieses Basiscurriculum soll das gemeinsame „gegenwärtige disziplinäre Selbstverständnis“ dokumentieren (Sektion BWP 2003: 4). Dieses Selbstverständnis ist aber ein mehrfach eingeschränktes ${ }^{14}$ :

(1) Es wird nur konkretisiert für die curriculare Konstruktion von universitären Berufsbildungsgängen für Berufs- und Wirtschaftspädagogen. ${ }^{15}$

(2) Es zielt auf die Professionalisierung pädagogischer Fachkräfte in der Berufsbildung, reflektiert aber nicht, dass die Professionalisierung nur Mittel zur Erreichung übergeordneter Ziele sein sollte. Denn die pädagogischen Fachkräfte sollen die ihnen anvertrauten Personen so fördern, dass sie in ihrer Umwelt von Berufsbildung und Berufsarbeit sich entwickeln können in Richtung auf eine berufsbildungstheoretisch begründeten Zielkategorie (z. B. „Berufsbildung“; vgl. Kell 2013: 10f.).

$12 \mathrm{Ob}$ diese kongressbezogene Stichprobe repräsentativ für die Forschungsaktivitäten von Sektionsmitgliedern zum Übergangsbereich sind, müsste geprüft werden. Hinzuweisen ist u.a. auf erkennbare Aktivitäten wie das Memorandum der Sektion ,zur Professionalisierung des pädagogischen Personals [...]“ (BWP 2009), auf die Forschungsbeiträge zur theoretischen und empirischen Fundierung des Memorandums, auf einen aktualisierten Überblick zur Forschungslage (Nickolaus 2012) und auf die Berichte über einschlägige Forschungen, die im Themenheft der Zeitschrift für Berufs- und Wirtschaftspädagogik (Heft 2, 2014) dokumentiert sind.

13 Wünschenswert wären Forschungen über die Wirkungen dieser Empfehlungen auf die Lehrangebote an allen Hochschulen mit Berufsschullehrerausbildung, auf die Kompetenzentwicklungen der Studierenden, die weitere Professionalisierung der Lehrkräfte, die Lehr-Lernprozesse in den beruflichen Schulen und letztendlich auf die Entwicklungen der lernenden Subjekte in der Berufsbildung.

14 Zur Selbstreflexion gehörte auch eine kritische Analyse der durch die Sektion organisierten Konsensfindungsprozesse und Vergleiche der Ergebnisse (siehe auch Fußnote 14).

15 Wenn bei curricularen Konstruktionen sechs Bezugspunkte berücksichtigt werden sollen: (1) Ziele für die Bildungsgänge; (2) Lernvoraussetzungen und Lernbedürfnisse der Adressaten (Subjekte); (3) Qualifikationsanforderungen von (Erwerbs)Berufen im systemischen Kontext von Strukturen und Entwicklungen der gesellschaftlichen Organisation von Arbeit; (4) Erkenntnisse der Wissenschaften; (5) organisatorische und institutionelle Umwelten für die Bildungsgänge; (6) Gestaltungsoptionen für berufsbezogenes Lehren und Lernen), dann ist die starke Fokussierung im Basiscurriculum auf den Bezugspunkt 4 und die Vernachlässigung des Bezugspunktes 1 deutlich zu erkennen (vgl. Kell 2013: 16). 
(3) Während in der Denkschrift zur Berufsbildungsforschung ein Selbstverständnis zur Zielkategorie Berufsbildung expliziert ist (vgl. DFG 1990: 62), fehlen vergleichbare Zielaussagen im Basiscurriculum. ${ }^{16}$

(4) Das von der Sektion 2009 verabschiedete „Memorandum zur Professionalisierung des pädagogischen Personals in der Integrationsförderung aus berufsbildungswissenschaftliche Sicht" ist bei der Fortschreibung des Basiscurriculums allenfalls marginal berücksichtigt. Im Leitbild wird lediglich bei den ,profilgebenden Vertiefungen“ auf (gleichrangige?) Schwerpunkte wie Inklusion, Übergangssystem. Entrepreneurship hingewiesen (Sektion BWP 2014: 7).

(5) Angemessene Folgerungen aus der Tatsache, dass seit 2009 „die Behindertenrechtskonvention (BKK) für die Bundesrepublik rechtsverbindlich“" ist und damit auch die Pädagogik und die Lehrerausbildung am Leitbild der Inklusion ausgerichtet werden muss (vgl. Allmendinger/ Warse 2014), sind für das Basiscurriculum noch nicht gezogen worden. Um die Zielkategorie Inklusion berufsbildungstheoretisch interpretieren zu können, fehlt noch die Konsensfindung zu einem das Basiscurriculum übergreifenden Selbstverständnis.

Abgesehen von den speziellen Problemen des Basiscurriculums und ergänzend $\mathrm{zu}$ der oben angesprochenen inhaltlichen Verständigung über das Selbstverständnis der BWP sollten auch Überlegungen zur sprachlichen Außendarstellung in den Konsensfindungsprozess einbezogen werden. Dazu folgende Überlegungen und Anregungen:

\subsection{Einheitliche Begriffe und Bezeichnungen statt Wortvielfalt}

Das Basiscurriculum wurde vorrangig erarbeitet für ein berufs- und wirtschaftspädagogisches (Teil)Studium (Studienfach) im Rahmen der akademischen Berufsausbildung für Lehrkräfte an beruflichen Schulen. Trotz klarer Aufgaben- und Funktionsbestimmung dieses Teilstudiums im Ausbildungs-

16 Ein positives Beispiel für die Präsentation eines wissenschaftlichen Selbstverständnisses: Die Gesellschaft für Arbeitswissenschaft (GfA), in der Wissenschaftler verschiedener Disziplinen organisiert sind, und die deshalb zwischen ihrer Selbstdarstellung als (Einheits)Disziplin „Arbeitswissenschaft“ oder als interdisziplinärer Forschungsbereich“ schwankt, hat bereits 1987 als übergreifendes Ziel die menschengerechte Gestaltung der Arbeit formuliert (vgl. Luczak/Volpert 1987: 56ff.). Auf der Homepage (www.gesellschaft-fuer-arbe itswissenschaft.de) hat die GfA ihr Selbstverständnis und Konkretisierungen in einem Memorandum dokumentiert. 
und Professionalisierungsprozess dieser Lehrkräfte sind die Bezeichnungen dafür von verwirrender Vielfalt. ${ }^{17}$

In den 1970er Jahren gab es dafür eine sachlich und sprachlich zutreffende Bezeichnung: „Erziehungs- und gesellschaftswissenschaftliches Teilstudium (EGT)“. Gegenwärtig verwendet die KMK „Bildungswissenschaften“ als Bezeichnung für dieses Teilstudium ${ }^{18}$ und ordnet damit Psychologie, Soziologie, Philosophie etc. den „Bildungswissenschaften“ $\mathrm{zu}$ - gegen deren eigenständiges disziplinäres Selbstverständnis. Da die KMK in den „Standards für die Lehrerbildung: Bildungswissenschaften“ (2004) offen lässt, welche Disziplinen es sind, "die sich mit Bildungs- und Erziehungsprozessen, mit Bildungssystemen sowie mit den Rahmenbedingungen auseinandersetzten“ (KMK 2004: 4) und mit welchen Anteilen (zeitlich, kapazitativ etc.) sie am Lehr- und Leistungskontingent beteiligt sein sollen, stehen die curricularen Konstruktionen für die „Bildungswissenschaften“ in der Spannung verschiedener disziplinärer Selbstverständnisse und unterschiedlicher hochschulpolitischer Interessen. In solchen Akteurkonstellationen sind die ortspezifischen Durchsetzungschancen des Basiscurriculums größer, wenn das darin dokumentierte gemeinsame Selbstverständnis offensiv vertreten und eine einheitliche Bezeichnung dafür verwendet wird. Deshalb sollte das Basiscurriculum

17 Zum Beleg der Bezeichnungsvielfalt für dieses Teilstudium weise ich nach Durchsicht von Lehrerbildungsgesetzen der Bundesländer, von gutachterlichen Expertisen und von einschlägigen Prüfungs- und Studienordnungen - ohne Anspruch auf Vollständigkeit und genauere Analysen - auf Folgendes hin: In 12 Bundesländern wurde die Bezeichnung Bildungswissenschaften in neueren Lehrerbildungsgesetzen bereits übernommen $(\mathrm{BW}, \mathrm{BE}$, BB, HB, MV, NI, RP, SL, SN, ST, SH, TH). Erziehungswissenschaftliches Studium (BY, NW), Erziehungswissenschaft einschließlich Fachdidaktik (Hamburger Modell) und Erziehungs- und Gesellschaftswissenschaften. (mit dem Schwerpunkt BWP) (HE) sind weitere Bezeichnungen in den Gesetzen. In Prüfungs- und Studienordnungen werden als Bezeichnungen verwendet: Erziehungswissenschaft(en) (mit dem Schwerpunkt Berufs- und/oder Wirtschaftspädagogik); Berufspädagogik; Wirtschaftspädagogik; bildungswissenschaftliche Anteile der BWP; das Fach Bildungswissenschaften; Professionalisierungsbereich (tragen alle anderen Studienanteile nicht zur Lehrerprofessionalisierung bei?); Berufswissenschaften; berufliche Bildungswissenschaften (sic!). Es bestehen Bezeichnungsunterschiede zwischen den Lehrerbildungsgesetzen der Länder und den Prüfungs- und Studienordnungen der landeseigenen Universitäten, zwischen den Ordnungen einzelner landeseigener Universitäten und auch zwischen denen innerhalb einer Universität, z. B. für „Gewerbelehrer“ (Berufspädagogik für Ingenieur- bzw. Technikpädagogen) und für „Handelslehrer“ (Wirtschaftspädagogik).

18 Durch die curricularen Konstruktionen des Teilstudiengangs „Bildungswissenschaften“ müssten auch die Wechselbeziehungen zu den Fachdidaktiken und zu den schulpraktischen Studien geklärt werden. Ein spezielles Problem für die Fachdidaktiken besteht in der Gestaltung der Spannung von Allgemein- und Berufsbildung. Am Beispiel ökonomischer Inhalte in allgemeinen Schulen (im Sachunterricht der Grundschule, in der Arbeitslehre/ Polytechnik im Sekundarbereich I, im Ökonomieunterricht im Gymnasium) als Teil einer vorberuflichen Bildung und denen in den Ausbildungsberufen des Berufsfeldes Wirtschaft und Verwaltung sind diese Spannungen zu erkennen. 
und das implizite disziplinäre Selbstverständnis der Ausgangspunkt sein für länder- und universitätsspezifische Konkretisierungen und Umsetzungen in den einschlägigen Studiengängen. Eine einheitliche Bezeichnung des Teilstudiengangs (Studienfachs) würde in der Außendarstellung das Gemeinsame betonen, die ortspezifischen Besonderheiten aber nicht ausschließen, sondern nur begründungspflichtig machen für Entscheidungen über Studien- und Prüfungsordnungen, Personal- und Sachmittel etc. In den Minderheitenpositionen, in denen sich die BWP in den Fachbereichen oder Fakultäten jeweils befindet, kann die Betonung des inhaltlich und sprachlich Gemeinsamen hilfreich sein. Die sprachliche Vielfalt und Vieldeutigkeit ist dagegen als eher hinderlich einzuschätzen.

\subsection{Singular statt Plural}

Erziehungswissenschaft (oder Bildungswissenschaft) ist eine wissenschaftliche Disziplin mit mehreren Teil- (oder Sub-)Disziplinen wie die BWP. Der Plural wird häufig für Fakultäten, Fachbereiche oder Institute verwendet, in denen Nachbarwissenschaften wie Psychologie und Soziologie vertreten sind. Dadurch werden diese Wissenschaften aber nicht zu einer Erziehungswissenschaft, sondern sie sollen in Studiengängen, die für pädagogische Berufe ausbilden, eigenständige - aber auf die pädagogische Praxis bezogene Beiträge liefern. Bei der curricularen Konstruktion von Studiengängen für pädagogische Berufe werden die Spannungen zwischen den verschiedenen disziplinären Selbstverständnissen deutlich, die nur konstruktiv überwunden und fruchtbar gemacht werden können, wenn im interdisziplinären Diskurs eine Vermittlung gelingt zwischen disziplinärer Eigenständigkeit und der komplexen Aufgabe einer Berufsbildung. Solche Zusammenhänge werden aber durch die Verwendung des Plurals verdeckt.

Auch die Verwendung des Plurals Berufsbildungswissenschaften ist nicht nur aus vergleichbaren Überlegungen verschleiernd, sondern trägt vielfach auch noch zur Verwischung der Unterschiede zwischen der Disziplin BWP und der interdisziplinären Berufsbildungsforschung bei. Dadurch wird das Besondere einer berufsbildungswissenschaftlich orientierten Berufsbildungsforschung, das durch die Orientierung an der Zielkategorie Berufsbildung konstituiert wird (vgl. van Buer/Kell 1999; Kell 2007), gegenüber z. B. einer psychologisch, soziologisch, wirtschaftswissenschaftlich etc. orientierten Berufsbildungsforschung vernachlässigt.

\subsection{Berufsbildungswissenschaft statt Berufs- und Wirtschaftspädago- gik}

Die Begriffe Wirtschaftspädagogik und Berufspädagogik haben sich im Wesentlichen im Kontext der Berufsschullehrerausbildung entwickelt. Die Wirtschaftspädagogik hat ihre Wurzeln in der 1898 in Leipzig begonnenen Grün- 
dung von Handelshochschulen, die im ersten Drittel des 20. Jahrhunderts durch Umwandlungen in Wissenschaftliche Hochschulen universitären Status erlangt haben. In den Wirtschafts- und Sozialwissenschaftlichen Fakultäten entwickelten sich die Studiengänge mit dem Abschluss Diplom-Handelslehrer. Die Ausbildung von Gewerbelehrern fand zeitlich etwa parallel in $\mathrm{Be}$ rufspädagogischen Instituten statt. Nach der Integration der Berufsschullehrerausbildung für alle (anderen) beruflichen Fachrichtungen in die Universitäten in den 1960er Jahren hat sich sprachlich für die Bezeichnung unserer wissenschaftlichen Disziplin und für die Organisation ihrer Wissenschaftlerinnen und Wissenschaftler eine additive Lösung durchgesetzt, die in der Doppelbezeichnung Berufs- und Wirtschaftspädagogik zum Ausdruck kommt.

Seit den 1960er Jahren ist in der BWP ein gemeinsames Selbstverständnis als erziehungswissenschaftliche (Teil-)Disziplin entwickelt worden. Ihr Gegenstandsbereich ist die Berufsbildung, ihr Erkenntnisinteresse ist fokussiert auf die Subjektentwicklung - in der Umwelt von Berufsbildung und Berufsarbeit - und ihre normative Orientierung schließt an den Bildungsbegriff der (allgemeinen) Erziehungswissenschaft an - mit speziellen berufsbildungstheoretischen Begründungen (z. B. zur Zielkategorie Berufsbildung; vgl. Kell 2007: Kapitel 3).

Auf den Gegenstandsbereich Berufsbildung sind auch weitere Begriffe wie Berufsbildungsforschung, -planung, -politik bezogen, so dass die Bezeichnung Berufsbildungswissenschaft auch mit dem allgemeinen Sprachgebrauch übereinstimmt. Zudem ist mit einer berufsbildungstheoretisch begründeten Berufsbildungswissenschaft auch ein Integrationsanspruch verbunden (vgl. Kell 2007: 99f.). Allerdings ist angesichts der großen Komplexität des Gegenstandsbereichs Berufsbildung eine Binnendifferenzierung der Berufsbildungswissenschaft erforderlich. Die sprachliche Zweiteilung in Berufsund Wirtschaftspädagogik bringt die faktische Binnendifferenzierung in der Berufsbildungswissenschaft aber nicht zum Ausdruck. ${ }^{19}$ Grundsätzlich geht es darum, in der Spannung von Einheit (Allgemeines) und Differenz (Spezielles) die Sektionsarbeit wissenschaftsorganisatorisch zu gestalten.

Als Bezugspunkt für eine Binnendifferenzierung einer Sektion Berufsbildungswissenschaft kommen die für die Berufschullehrerausbildung von der KMK festgelegten 16 Beruflichen Fachrichtungen in Betracht (vgl. KMK 2013). Sie können als Reaktion auf die berufliche Organisation der Arbeit im Beschäftigungssystem interpretiert werden und stehen im Kontext mit Bemühungen um die Klassifikation von Berufen, um Berufsfeldschneidungen, Berufsgruppenbildungen etc. (vgl. Lipsmeier 2014). Da für die Beruflichen

19 In der DDR bestand eine Differenzierung in Agrar-, Medizin- und Wirtschaftspädagogik (an der HU-Berlin) und in Ingenieurpädagogik (für die gewerblich-technischen Fachrichtungen an der TU-Dresden) (vgl. Kell 2011: 45. 
Fachrichtungen Berufs/Fach-Didaktiken zu entwickeln sind, bietet sich eine Binnendifferenzierung der Sektion Berufsbildungswissenschaft in berufs/ fachdidaktische Kommissionen an. Eine in der Sektion organisierte Zusammenarbeit in solchen Kommissionen könnte für diese permanente curriculare Entwicklungsarbeit förderlich sein. Zur Verfolgung der Integrationsperspektive sollte eine weitere (Querschnitt)Kommission „Benachteiligte“ organisiert werden.

Mit dem Vorschlag, die Sektion nicht als Berufs- und Wirtschaftspädagogik, sondern als Berufsbildungswissenschaft zu bezeichnen, wird mit dieser Bezeichnung auch eine Entwicklung nachgeholt, die bei der Gründung der DGfE vor 50 Jahren zur sprachlichen Differenzierung zwischen Pädagogik als gesellschaftliche Praxis und Erziehungswissenschaft als universitäre Disziplin geführt hat.

\section{Schlussbemerkung}

Als Emeritus mit einiger Distanz zur anspruchsvollen und anstrengenden Berufsarbeit in der Berufsausbildung pädagogischer Fachkräfte und entlastet von Außensteuerungen durch politische Vorgaben, Akkreditierungen, Evaluationen, Zertifizierungen etc. habe ich mehr Zeit und Gelegenheit, aus der Vogelperspektive über Entwicklungen in der „Zunft“ nachzudenken. Zum Nachdenken fühle ich mich als Emeritus aber auch verpflichtet - in Einsamkeit und Freiheit. Vielleicht können meine Überlegungen die Mitglieder der Sektion Berufs- und Wirtschaftspädagogik dazu anregen, über das Selbstverständnis, Genauigkeit der Begriffe, organisatorische Binnendifferenzierungen etc. individuell nachzudenken und gemeinsam zu beraten.

\section{Literatur}

Allmendinger, Jutta/Wrase, Michael (2014): Das Recht auf Inklusion. Schulsystem und Pädagogik müssen sich ändern. In: WZB Mitteilungen, 145 S. 39-42.

Arnold, Rolf/Lipsmeier, Antonius (Hrsg.) (2006): Handbuch der Berufsbildung. 2., überarbeitete und ergänzte Auflage. Wiesbaden: VS Verlag für Sozialwissenschaften.

Behm, Britta (2014): 50 Jahre „Deutsche Gesellschaft für Erziehungswissenschaft" (DGfE). Gedanken zu Jubiläum und Forschungslücken. In: Erziehungswissenschaft 25, 48, S. 11-23.

Buchmann, Ulrike/Bylinski, Ursula (2013): Ausbildung und Professionalisierung von Fachkräften für eine inklusive Berufsbildung. In: Döbert, H./ Weishaupt, H. (Hrsg.): Inklusive Bildung professionell gestalten. Situati- 
onsanalyse und Handlungsempfehlungen. Münster u.a.: Waxmann, S. 147-202.

Büchter, Karin/Klusemeyer, Jens/Kipp, Martin (Hrsg.) (2009): Selbstverständnis der Disziplin Berufs- und Wirtschaftspädagogik. www.bwpat.de /content/ausgabe/16/ [Zugriff: 15. Oktober 2014].

Buer, Jürgen van/Kell, Adolf (1999): Forschungsprojekt „Berichterstattung über Berufsbildungsforschung“. Abschlußbericht. (Arbeitsgemeinschaft Berufsbildungsforschungsnetz AG BFN). Berlin, Siegen: Humboldt-Universität.

Bylinski, Ursula (2014): Übergangsgestaltung als neue Anforderung an die Professionalität der pädagogischen Fachkräfte. In: Zeitschrift für Berufsund Wirtschaftspädagogik 110, 2, S. 235-256.

Deutsche Forschungsgemeinschaft (Hrsg.) (1990): Berufsbildungsforschung an den Hochschulen der Bundesrepublik Deutschland. Denkschrift. Weinheim: VCH Acta humanoria.

Deutsche Gesellschaft für Erziehungswissenschaft (2012): Programmheft zum 34. Kongress. http://www.dgfe2012.de/ [Zugriff: 15. Oktober 2014].

Deutsche Gesellschaft für Erziehungswissenschaft (2014): Programmheft zum 24. Kongress. http://www.dgfe2014.de/wp-content/uploads/DGfE_P rogramm2014_K26Web.pdf [Zugriff: 15. Oktober 2014].

Deutsche UNESCŌ-Kommission (DUK) (Hrsg.) (2009): Inklusion: Leitlinien für die Bildungspolitik. Bonn: Deutsche UNESCO-Kommission.

Döbert, Hans/Weishaupt, Horst (Hrsg.) (2013): Inklusive Bildung professionell gestalten. Situationsanalyse und Handlungsempfehlungen. Münster u.a.: Waxmann.

Heid, Helmut (1987): Zur Situation der Erziehungswissenschaft in der Bundesrepublik Deutschland. In: Zeitschrift für internationale erziehungsund sozialwissenschaftliche Forschung 4, 2, S. 225-251.

Huisinga, Richard/Lisop, Ingrid (1999): Wirtschaftspädagogik. München: Vahlen.

Kaiser, Franz-Josef/Pätzold, Günter (Hrsg.) (2006): Wörterbuch Berufs- und Wirtschaftspädagogik. Zweite, überarbeitete und erweiterte Auflage. Bad Heilbrunn: Klinkhardt.

Kell, Adolf (2006): Organisation, Recht und Finanzierung der Berufsbildung. In: Arnold, R./Lipsmeier, A. (Hrsg.): Handbuch der Berufsbildung. Zweite Auflage. Wiesbaden: VS Verlag für Sozialwissenschaften, S. 453-484.

Kell, Adolf (2007): Ökologisch orientierte Berufsbildungswissenschaft - Eine berufsbildungstheoretische Positionierung. In: Greb, U./Schüßler, I. (Hrsg.): Berufliche Bildung als nachhaltige Ressource. Frankfurt am Main: Verlag G.F.A.B., S. 87-116.

Kell, Adolf (2010): Berufsbildungsforschung: Gegenstand, Ziele, Forschungsperspektiven. In: Nickolaus, R./Pätzold, G./Reinisch, H./Tramm, 
T. (Hrsg.): Handbuch Berufs- und Wirtschaftspädagogik. Bad Heilbrunn: Klinkhardt, S. 355-367.

Kell, Adolf (2011): Berufsschullehrerbildung zwischen Politik und Wissenschaft. In: Zlatkin-Troitschanskaia, O. (Hrsg.): Stationen empirischer Bildungsforschung. Traditionslinien und Perspektiven. Wiesbaden: VS Verlag für Sozialwissenschaften, S. 439-462.

Kell, Adolf (2013): Produktionsschule - Übergangssystem - Lern-ArbeitsSystem: Berufsbildungswissenschaftliche Perspektiven. In: bwp@ Spezi al 6 - Hochschultage Berufliche Bildung 2013, Workshop 09, hrsg. v. Ge ntner, C./Meier J., 1-34. http://www.bwpat.de/ht2013/ws09/kell_ws09-ht 2013.pdf [Zugriff: 5. Oktober 2014].

Lipsmeier, A. (2014): Vom Berufsfeld zur Berufsgruppe - bloße Umbenennung oder Innovation? In: Zeitschrift für Berufs- und Wirtschaftspädagogik 110, 2, S. 295-304.

Luczak, Holger/Volpert, Walter et al. (1987): Arbeitswissenschaft. Kerndefinition - Gegenstandskatalog - Forschungsgebiete. Eschborn: RKW.

Müller, Hans-Rüdiger/Bohn, Sabine/Thole, Werner (Hrsg.) (2013): Erziehungswissenschaftliche Grenzgänge. Markierungen und Vermessungen. Opladen u.a.: Verlag Barbara Budrich.

Nickolaus, Reinhold (2012): Erledigen sich die Probleme an der ersten Schwelle von selbst? Strukturelle Probleme und Forschungsbedarfe. In: Zeitschrift für Berufs- und Wirtschaftspädagogik 1008, 1, S. 5-17.

Nickolaus, Reinhold/Pätzold, Günter/Reinisch, Holger/Tramm, Tade (Hrsg.) (2010): Handbuch Berufs- und Wirtschaftspädagogik. Bad Heilbrunn: Klinkhardt.

Niedermair, Gerhard (Hrsg.) (2013): Facetten berufs- und wirtschaftspädagogischer Forschung. Linz: Trauner.

Rauner, Felix (Hrsg.) (2005): Handbuch Berufsbildungsforschung. Bielefeld: W. Bertelsmann.

Sektion Berufs- und Wirtschaftspädagogik der Deutschen Gesellschaft für Erziehungswissenschaft (2003): Basiscurriculum für das universitäre Studienfach Berufs- und Wirtschaftspädagogik (Beschlossen von der Mitgliederversammlung in Oldenburg am 25. März 2003). http://www.b wp-dgfe.de/images/Dokumente/Basiscurriculum_BWP_040202.pdf [Zugriff: 15. Oktober 2014].

Sektion Berufs- und Wirtschaftspädagogik der Deutschen Gesellschaft für Erziehungswissenschaft (Hrsg.) (2009): Memorandum zur Professionalisierung des pädagogischen Personals in der Integrationsförderung aus berufsbildungswissenschaftlicher Sicht. Bonn: Pahl-Rugenstein.

Sektion Berufs- und Wirtschaftspädagogik der Deutschen Gesellschaft für Erziehungswissenschaft (2014): Basiscurriculum für das universitäre Studienfach Berufs- und Wirtschaftspädagogik im Rahmen berufs- und wirtschaftspädagogischer Studiengänge Wirtschaftspädagogik (Beschlos- 
sen von der Mitgliederversammlung in Schwäbisch Gmünd am 25. September 2014). http://www.bwp-dgfe.de/sektion/positionen/curriculum/ [Zugriff: 15. Oktober 2014].

Sloane, Peter F. E./Twardy, Martin/ Buschfeld, Detlef (2004): Einführung in die Wirtschaftspädagogik. Zweite Auflage. Paderborn: Eusl.

Ständige Konferenz der Kultusminister der Länder (KMK) (2004): Standards für die Lehrerbildung: Bildungswissenschaften (Beschluss vom 16.12. 2004). www.kmk.org/fileadmin/veroeffentlichungen_beschluesse/2004/2 004_12_16-Standards_leherbildung.pdf [Zugriff: 15. Oktober 2013].

Ständige Konferenz der Kultusminister der Länder (KMK) (2013): Rahmenvereinbarung über die Ausbildung und Prüfung für ein Lehramt der Sekundarstufe II (berufliche Fächer) oder für die beruflichen Schulen (Lehramtstyp 5) (vom 12.05.1995 i. d. F. vom 07.03.2013) www.kmk.or g/fileadmin/veroeffentlichungen_beschluesse/1995/1995_05_12-RV_Le hramtstyp-5 pdf [Zugriff: 15. Oktober 2014].

Tippelt, Rudolf/Schmidt-Hertha, Bernhard (2013): Inklusion im Hochschulbereich. In: Döbert, H./Weishaupt, H. (Hrsg.) (2013): Inklusive Bildung professionell gestalten. Situationsanalyse und Handlungsempfehlungen. Münster u.a.: Waxmann, S. 203-229.

Zabeck, Jürgen (2013): Geschichte der Berufserziehung und ihrer Theorie. Zweite Auflage. Paderborn: Eusl. 